\title{
Protective Effects of Honey-Processed Astragalus on Liver Injury and Gut Microbiota in Mice Induced by Chronic Alcohol Intake
}

\author{
Jingxuan Zhou $\mathbb{D}^{1},{ }^{1}$ Nanhai Zhang $\mathbb{D}^{1},{ }^{1}$ Liang Zhao $\mathbb{D}^{2},{ }^{2}$ Mohamed Mohamed Soliman $(\mathbb{D}){ }^{3}$ \\ Wei Wu, ${ }^{4}$ Jingming Li, ${ }^{5}$ Feng Zhou $\mathbb{D}^{1},{ }^{1}$ and Liebing Zhang $\mathbb{D}^{1}$ \\ ${ }^{1}$ Beijing Key Laboratory of Functional Food from Plant Resources, College of Food Science and Nutritional Engineering, \\ China Agricultural University, 17 Tsinghua East Road, Beijing 10083, China \\ ${ }^{2}$ Beijing Engineering and Technology Research Center of Food Additives, Beijing Technology and Business University (BTBU), \\ 11 Fucheng Road, Beijing 100048, China \\ ${ }^{3}$ Clinical Laboratory Sciences Department, Turabah University College, Taif University, Taif, Saudi Arabia \\ ${ }^{4}$ College of Engineering, China Agricultural University, 17 Tsinghua East Road, Beijing 10083, China \\ ${ }^{5}$ Center for Viticulture and Enology, College of Food Science and Nutritional Engineering, China Agricultural University, \\ 17 Tsinghua East Road, Beijing 10083, China
}

Correspondence should be addressed to Feng Zhou; zf@cau.edu.cn and Liebing Zhang; lbzhang@cau.edu.cn

Received 25 October 2021; Accepted 22 December 2021; Published 11 January 2022

Academic Editor: Rana Muhammad Aadil

Copyright ( 92022 Jingxuan Zhou et al. This is an open access article distributed under the Creative Commons Attribution License, which permits unrestricted use, distribution, and reproduction in any medium, provided the original work is properly cited.

\begin{abstract}
Honey-processed Astragalus (HPA) is a mixture of Astragalus and honey, which is a processed product of Chinese medicine. It has the active ingredients of Astragalus and the unique effects of honey. However, the mechanism of HPA for improving alcoholic liver disease (ALD) is not clear. The purpose of this study is to explore the ameliorating effect and mechanism of HPA ( 4 and $8 \mathrm{~g} / \mathrm{kg}$ bw) on alcoholic liver injury. Two doses of HPA were orally administered to alcohol-treated mice for four weeks. The results showed that HPA could effectively reduce triglycerides (TG) by $59 \%$ and free fat acid (FFA) and total cholesterol (TC) in serum and hepatic were reduced by least $25.9 \%$. HPA could cause a decrease in serum low-density lipoprotein cholesterol (LDL-C) from $0.145 \mathrm{mM}$ to $0.117 \mathrm{mM}$, and the serum high-density lipoprotein cholesterol (HDL-C) was increased. After alcohol-treated mice were supplemented with HPA, antioxidant markers (superoxide dismutase (SOD), catalase (CAT), glutathione (GSH), and Glutathione peroxidase (GSH-Px)), liver function index (alanine aminotransferase (ALT), aspartate aminotransferase (AST), and alkaline phosphatase (ALP)), proinflammatory cytokines (tumor necrosis factor- $\alpha$ (TNF- $\alpha$ ), interleukin- 6 (IL-6), and interleukin$1 \beta$ (IL-1 $\beta)$ ), and liver tissue were all significantly improved. This is related to the fact that HPA can promote the expression of oxidative stress-related genes and inhibit the expression of inflammation-related genes. In addition, HPA could also regulate the disturbance of the intestinal microflora. In general, HPA could significantly improve the accumulation of serum and liver lipids caused by alcohol and the imbalance of intestinal flora in mice. It could also improve liver function, oxidative stress, and inflammation.
\end{abstract}

\section{Introduction}

Alcohol-related diseases are one of the most common preventable diseases in the world [1]. More than 3 million people around the world die from alcoholism every year [2]. Alcohol injury can cause damage to many end-organs and systems in the body, and alcoholic liver disease (ALD) is an important manifestation of liver injury [3]. ALD can develop from asymptomatic to alcoholic fatty liver, liver cirrhosis, and even alcoholic hepatitis and hepatocellular carcinoma $[2,4]$. There is evidence that the development mechanism of ALD is closely related to oxidative stress, inflammatory development, and intestinal microflora disorders [5]. Although drugs such as disulfiram, naltrexone, and corticosteroids are used in abstinence treatment, some drugs are not approved for ALD treatment. Corticosteroids are effective only in the short term in $50 \%$ patients with ALD $[6,7]$. Due to the personal differences of patients and adverse reactions 
caused by drugs still exist, so it is common to choose natural products with low side effects and good tolerance to achieve the improvement of ALD.

Astragalus (Huangqi, Astragalus membranaceus Beg. var. mongholicus (Beg.)) appears in people's field of vision as a common healthcare traditional Chinese medicine because of its antioxidant, anti-inflammatory, and other biological activities [8-10]. Astragalus extracts can improve lipopolysaccharide-induced liver injury by inhibiting the formation of lipid peroxides and proinflammatory factors [11]. Our previous studies showed that Astragalus polysaccharides and Astragalus saponins could improve liver injury in ALD mice [12]. As a safe alternative sweetener, honey could reduce triglycerides (TG), total cholesterol (TC), and lowdensity lipoprotein cholesterol (LDL-C) [13]. Data showed that honey could improve liver injury by regulating oxidative stress markers: catalase (CAT), glutathione (GSH), malondialdehyde (MDA), superoxide dismutase (SOD), and liver function $[14,15]$. Honey-processed Astragalus (HPA) is a mixture of astragalus and honey [16]. Some studies have shown that after the combination of honey and Astragalus, the structure of the active components of Astragalus has changed, and the efficacy of HPA was stronger than that of Astragalus $[16,17]$. In addition, HPA had a certain antiinflammatory effect $[16,17]$. HPA could not only improve the inflammatory response but also regulate the gut microbial flora diversity of colitis mice [18].

However, the mechanism and research of HPA alleviating ALD are still poorly understood. In view of the above biological activities of HPA, we speculate that HPA may have a beneficial effect on improving ALD. Therefore, this study aims to evaluate the improvement effect of HPA (Hunyuan, Shanxi Province) on alcohol-induced liver injury in mice and the effect of reducing serum and hepatic lipids. In addition, explore the mechanism of inhibiting oxidative stress, inflammation, and the influence of intestinal flora in alcohol-treated mice.

\section{Materials and Methods}

2.1. Materials and Chemicals. Honey-processed Astragalus was provided by Ze Qingqi Industry Development Co., Ltd. (Shanxi, China). Edible alcohol was purchased from Henan Xinheyang Alcohol Co., Ltd. (Henan, China). The assay kits of CAT, SOD, GSH, MDA, $\gamma$-glutamyl transpeptidase $(\gamma$-GT), glutathione peroxidase (GSH-Px), and free fat acid (FFA) and the commercial enzyme-linked immunosorbent assay (ELISA) kits of tumor necrosis factor- $\alpha$ (TNF- $\alpha$ ), interleukin-6 (IL-6), and interleukin-1 $\beta$ (IL-1 $\beta$ ) were bought from Beijing Sinouk Institute of Biological Technology (Beijing, China). The commercial assay kits of TG, TC, highdensity lipoprotein cholesterol (HDL-C), LDL-C, alanine aminotransferase (ALT), aspartate aminotransferase (AST), and alkaline phosphatase (ALP) were purchased from Biosino Bio-Technology and Science Inc. (Beijing, China). FastQuant RT Kit and SuperReal PreMix Plus with SYBR Green were purchased from Tiangen Biotech Co. Ltd. (Beijing, China). E.Z.N.A. ${ }^{\circledR}$ soil DNA Kit was bought from Omega Bio-tek (Norcross, GA, USA). AxyPrep DNA Gel
Extraction Kit was provided by Axygen Biosciences (Union City, CA, USA). Other reagents, such as ethanol, hydrochloric acid, chloroform, acetone, and $\mathrm{NaOH}$, were all of analytical grade.

2.2. Animals and Experimental Design. Forty-eight 5-weekold male ICR mice ( $20 \pm 1 \mathrm{~g}$ of weight) were all bought from Beijing Vital River Laboratory Animal Technology Co., Ltd. (Beijing, China; Certificate no. SCXK (Beijing) 2016-0006). The mice were kept in separate cages with fed food and water ad libitum. The mice were housed in a controlled environment with $40-55 \%$ humidity and a $12 \mathrm{~h}$ light/dark cycle at $22-24^{\circ} \mathrm{C}$. All animal procedures act up to the Animal Ethics Committee of the Beijing Key Laboratory of Functional Food from Plant Resources and the guidelines for the care and use of laboratory animals of the National Institutes of Health.

All mice were randomly divided into normal group (NG), model group (MG), HPA low-dose group (HPAL), and HPA high-dose group (HPAH) after adaption for 1 week ( $n=12$ each group). The mice in the NG and MG were orally distilled water for the following 4 weeks. After 1 hour of treatment with distilled water, the MG were given orally $10 \mathrm{~mL} / \mathrm{kg}$ body weight (bw) of $50 \%$ alcohol. In the HPAL and HPAH, HPA was formulated into a solution with a mass fraction of $40 \%$ and $80 \%$ (HPA was dissolved in distilled water). First, all mice were given orally the same dose alcohol as MG. Then, the HPAL and HPAH were administrated with $40 \%$ and $80 \%$ of HPA solution by oral route respectively $(10 \mathrm{~mL} / \mathrm{kg} \mathrm{bw})$ after treatment with $50 \%$ alcohol 1 hour later. The mice were weighted every three days, and the perfusion volume was adjusted based on the body weight. After the fourth week of feeding, the mice were weighed and recorded for the last time. The mice were fasted for 12 hours with water freely before the blood samples were taken. The blood samples were taken from the orbital venous plexus of the mice and stored at $4^{\circ} \mathrm{C}$ for 12 hours. The liver tissue was weighed immediately after the mice were killed and the liver index was calculated [liver index (\%) = liver weight (g)/final body weight $(\mathrm{g}) \times 100 \%]$. The liver sample was divided into two parts: one part was immersed in $10 \%$ formalin solution for histopathology; the other part was used for biochemical determination and stored at $-80^{\circ} \mathrm{C}$. Colon contents were collected and stored at $-80^{\circ} \mathrm{C}$ for intestinal microbiota analysis.

\subsection{Analysis of Biochemical Indicators in Serum and Hepatic.} The serum was obtained after the blood samples centrifuged at $4^{\circ} \mathrm{C}$ with $4000 \mathrm{~g}$ for 15 minutes. According to the test kits, the levels of serum TC, TG, FFA, HDL-C, LDL-C, AST, ALT, APL, and $\gamma$-GT were tested by the Mindray BS-420 automatic biochemical analyzer (Shenzhen Mindray Biomedical Electronics Co., Ltd.). According to the method of Zhao et al. [19], the liver sample was homogenized, and then liver lipids were extracted from liver homogenate. The hepatic TC, TG, and FFA were determined by the same corresponding detection kits for detection with serum TC, TG, and FFA. The activities of SOD, CAT, GSH, GSH-Px, and the level of MDA 
in liver were detected using the corresponding assay kits. The contents of TNF- $\alpha$, IL- 6 , and IL- $1 \beta$ were measured following the instructions of the corresponding ELISA kits. The BCA kit was used to measure the concentration of total protein in liver homogenate.

2.4. Analysis of Real-Time PCR. The total liver RNA was extracted by using TRIpure reagent according to the instruction and reverse-transcribed into cDNA by using FastQuant RT Kit. The levels of mRNA expression were measured according to the kit manufacturer's instruction by SuperReal PreMix Plus with SYBR Green [20]. Glyceraldehyde-3-phosphate dehydrogenase (GAPDH) as the internal reference was measured to normalize mRNA expression. The appropriate primers are shown in Table 1. The relative mRNA expression was calculated by the $2^{-\Delta \Delta \mathrm{Ct}}$ method (relative expression $=2^{-\Delta \Delta \mathrm{Ct}}(\Delta \mathrm{Ct}$ (test) $=\mathrm{Ct}$ (target, test) $-\mathrm{Ct}$ (ref, test), $\Delta \mathrm{Ct}$ (calibrator) $=\mathrm{Ct}$ (target, calibrator) $-\mathrm{Ct}$ (ref, calibrator), $\Delta \Delta \mathrm{Ct}=\Delta \mathrm{Ct}$ (test) $-\Delta \mathrm{Ct}$ (calibrator))) [21].

2.5. Histological Analysis. The extracted livers were fixed in $10 \%(\mathrm{v} / \mathrm{v})$ formalin solution for $24 \mathrm{~h}$, before the samples were cut into slices. Then, the slices were embedded in paraffin. The slices were stained with hematoxylin and eosin (H\&E) and Masson. Finally, the sections were placed under a light microscope (BA-9000, Osaka, Japan) for observation.

2.6. Analysis of Gut Microbiota. The genome of fecal flora was extracted by using the DNA extraction kit, and the DNA samples were determined by NanoDrop2000 spectrophotometer (Thermo Fisher Scientific, Waltham, MA, USA). The V3-V4 region of the bacterial 16S rDNA genes was amplified using the barcoded universal primers (338F: $5^{\prime}$ ACTCCTACGGGAGGCAGCAG-3'; 806R: 5'-GGACTACHVGGGTWTCTAAT- $\left.3^{\prime}\right)$. The amplified products were confirmed by agarose gel electrophoresis for completeness and purified by AxyPrep DNA Gel Extraction Kit. The amplified products library was paired-end-sequenced on Illumina Miseq platform (Illumina, San Diego, CA, USA). Gut microbiota analysis was completed by Majorbio Bio-Pharm Technology Co., Ltd. (Shanghai, China).

2.7. Statistical Analysis. The results of the animal experiment were presented as mean \pm standard deviation (SD). SPSS 25.0 was used to evaluate the statistical analysis between model and other groups by independent-samples $T$ test using SPSS 25.0 (SPSS Inc., Chicago, USA). The figures were drawn by GraphPad Prism version 8.0 (La Jolla, CA, USA). $p<0.05$ was recognized as statistically significant and $p<0.01$ were regarded as highly statistically significant.

\section{Result}

3.1. Effect of HPA on Food Intake, Body Weight, and Hepatic Index in ALD Mice. As shown in Table 2, the final body weight and food intake of alcohol-treated mice were significantly lower than the NG $(p<0.01)$. The hepatic index in the MG was lower than that in the NG $(p<0.05)$. Only the high-dose HPA could clearly decrease the hepatic index compared to the MG $(p<0.05)$.

3.2. Effect of HPA on Lipids of Serum and Hepatic in ALD Mice. Figures 1(a)-1(e) present the influence of HPA on the serum lipids (TC, TG, FFA, HDL-C, and LDL-C). Compared with the NG, the levels of serum of TC, TG, FFA, and LDL-C in alcohol-treated mice were apparently increased $(p<0.01)$. Serum HDL-C in the MG was significantly reduced by $25.3 \%$ (versus NG, $p<0.01$ ). As displayed in Figures $1(\mathrm{f})-1(\mathrm{~h})$, the contents of hepatic lipids (TC, TG, and FFA) in the MG were obviously higher than that in the NG $(p<0.01)$. The levels of serum and hepatic lipids in all treatment groups were improvement apparently (versus MG, $p<0.01$ ). The contents of serum TG and hepatic TG in the HPAH could reduce by more than 59\% (versus MG, $p<0.01$ ). As Figures $1(\mathrm{a})-1(\mathrm{~h})$, the high-dose HPA had the best improvement effect on the lipids of serum and hepatic in ALD mice.

3.3. Effect of HPA on Hepatic Function in ALD Mice. Figure 2 shows that the activities of ALT, AST, ALP, and $\gamma$-GT in the MG were apparently higher than that in the NG $(p<0.01)$. The HPA could reduce the levels of these indexes (versus MG, $p<0.01$ ). In particular, the improvement effect of high dose HPA was the best, reducing AST (18.82\%), ALT (23.67\%), ALP (31.76\%), and $\gamma$-GT (26.66\%), respectively.

3.4. Effect of HPA on the Hepatic Oxidative Stress in ALD Mice. As shown in Figures 3(a)-3(e), there was a significant plummet in the CAT, SOD, GSH, and GSH-Px contents that occurred in alcohol-treated mice, and the MDA level had an apparently elevation (versus NG, $p<0.01$ ). After treatment with HPA, the content of oxidation markers in the liver of ALD mice was significantly improved (versus MG, $p<0.01$ ). In terms of SOD, GSH-Px, and MDA, the improvement effect of the HPAH was better than that of the HPAL. Especially, high dose of HPA could reduce the MDA level in ALD mice to $60 \%$ of the original (Figure $3(\mathrm{e}), p<0.01$ ). In addition, HPAL had the best improvement effect on CAT and GSH, and low dose of HPA could increase the level of CAT in ALD mice by 0.27 times (versus MG, $p<0.01$ ).

3.5. Effect of HPA on Inflammation Response in ALD Mice. As shown in Figures 4(a)-4(c), the effect of HPA on the level of inflammatory factors in ALD mice was evaluated. The level of inflammatory factors (IL-1 $\beta$, IL-6, and TNF- $\alpha$ ) increased significantly in alcohol-treated mice (versus NG, $p<0.01$ ). The three indexes in the treatment groups were obviously decreased (versus MG, $p<0.01$ ). In the levels of IL- $1 \beta$ and TNF- $\alpha$, the HPAH had the best improvement effect. The HPAH not only reduced the values of IL- $1 \beta$ from 7.77 to $3.86 \mathrm{pg} / \mathrm{mg}$ pro but also reduced the TNF- $\alpha$ index by $4.12 \mathrm{pg} / \mathrm{mg}$ pro (versus MG, $p<0.01$ ). Compared with the MG, the content of IL- 6 in the HPAL was only 0.67 times that in the MG (Figure 4(b)). 
TABLE 1: Primer sequences of genes used for qRT-PCR.

\begin{tabular}{lcc}
\hline Gene & Forward primer $\left(5^{\prime}-3^{\prime}\right)$ & Reverse primer $\left(5^{\prime}-3^{\prime}\right)$ \\
\hline Gapdh & TCTCCTGCGACTTCAACA & TGTAGCCGTATTCATTGTCA \\
Keap 1 & CAGATTGACAGCGTGGTT & GCAGTGTGACAGGTTGAA \\
$N f e 212$ & GTGCTCCTATGCGTGAAT & TCTTACCTCTCCTGCGTATA \\
Hmox 1 & AGGTCCTGAAGAAGATTGC & TCTCCAGAGTGTTCATTCG \\
Nqo 1 & ATGAAGGAGGCTGCTGTA & AGATGACTCGGAAGGATACT \\
$M y d 88$ & TGACATTCCTTCTTCAACCA & CACAGCCACCAGATTCTC \\
$N f k b 1$ & CCGTGAGGATATACTGAAGG & TTAAGCCGATAGTCTGTCTG \\
\hline
\end{tabular}

TABLE 2: Effects of AP and AS on body weight, food intake, and liver index of mice.

\begin{tabular}{lcccc}
\hline Groups & Initial weight $(\mathrm{g})$ & Final weight $(\mathrm{g})$ & Food intake $(\mathrm{g} / \mathrm{d})$ & Hepatic index $(\%)$ \\
\hline NG & $28.38 \pm 1.17$ & $34.09 \pm 2.97$ & $4.92 \pm 0.46$ & $3.63 \pm 0.22$ \\
MG & $28.55 \pm 1.49$ & $28.98 \pm 2.00^{* *}$ & $3.63 \pm 0.66^{* *}$ & $3.99 \pm 0.11^{*}$ \\
HPAL & $28.90 \pm 1.69$ & $28.98 \pm 1.17$ & $3.28 \pm 0.73$ & $3.82 \pm 0.16$ \\
HPAH & $28.74 \pm 1.32$ & $28.63 \pm 1.98$ & $3.20 \pm 0.68$ & $3.73 \pm 0.22^{\#}$ \\
\hline
\end{tabular}

Data were expressed as mean \pm SD $(n=12) .{ }^{* *} p<0.01$ and ${ }^{*} p<0.05$, versus NG; ${ }^{\#} p<0.05$, versus MG.

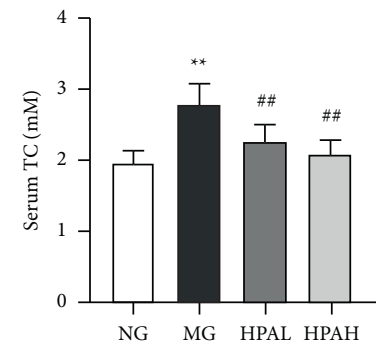

(a)

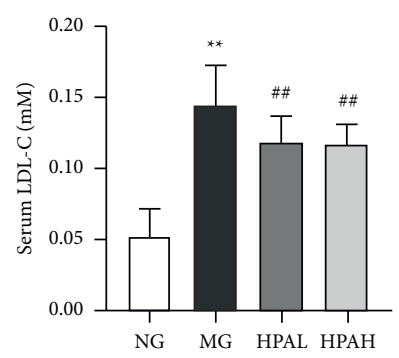

(e)

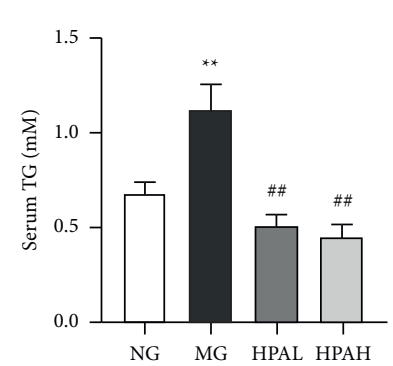

(b)

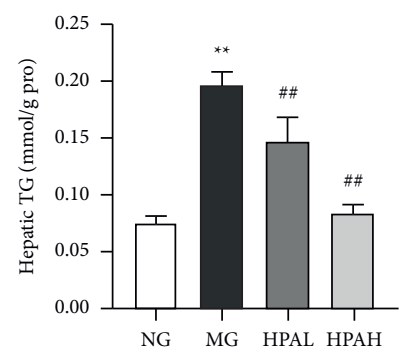

(f)

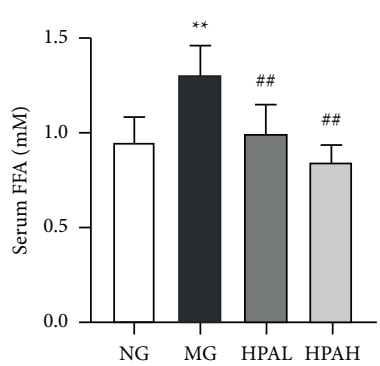

(c)

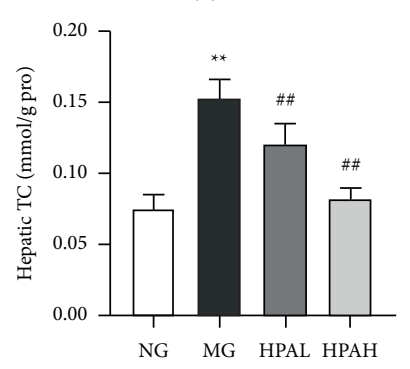

(g)

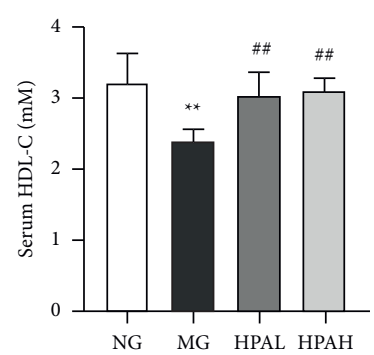

(d)

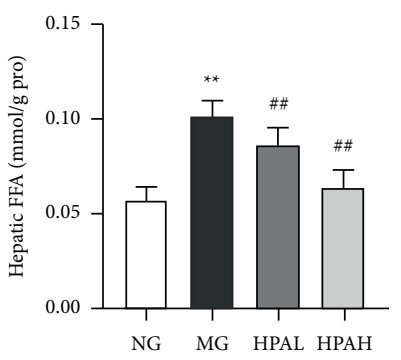

(h)

FIgUre 1: Effect of HPA on the serum and liver lipids in ALD mice. (a) Serum TG; (b) serum TC; (c) serum HDL-C; (d) serum LDL-C; (e) serum FFA; (f) hepatic TG; (g) hepatic TC; (h) hepatic FFA. Data were expressed as mean \pm SD $(n=12) .{ }^{* *} p<0.01$, versus NG; ${ }^{*} p<0.05$ and ${ }^{\# \#} p<0.01$, versus $M G$.

3.6. Effect of HPA on Oxidative Stress and InflammationRelated Gene Expression in ALD Mice. Figure 5 shows the effect of HPA on gene expression in inflammatory signal pathway (TLR4 (Tlr4), Myd88 (Myd88), and NF- $\kappa \mathrm{B}(N f k b 1)$ ) and oxidative stress signal pathway (Kelch-like ECH-associated protein 1 (Keap1 (Keap1)), NF-E2-related factor 2 (NRF2 $(\mathrm{Nfe} 2 \mathrm{l} 2))$, heme oxygenase 1 (HO-1 (Hmox 1$))$, and NADPH quinone dehydrogenase 1 (NQO1 (Nqo1)) in alcohol-treated mice. After the mice were treated by alcohol, the mRNA expression of Keap1, Nfe2l2, Hmox1, and Nqo1 was significantly downregulated, while the mRNA expression of Tlr4, Myd88, and $N f k b 1$ was significantly upregulated (versus NG, $p<0.01$ ). It has been observed that the HPA could obviously reduce the expression of Keap1, Nfe2l2, Hmox1, and Nqo1 and clearly increased the expression of Tlr4, Myd88, and Nfkb1 (vs. MG, $p<0.01)$. Compared with the MG, the genes related to oxidative stress in the HPAH increased more than 2 times, and the genes related to inflammation in the HPAH decreased by more than $36.6 \%$. Therefore, it can be seen that HPAH reduced the expression of inflammation-related genes and at the same time promotes the expression of antioxidant-related genes.

\subsection{Effect of AP and AS on Histopathological Variations of} Livers in ALD Mice. In order to confirm that the HPA has improvement effect on ALD, hepatic sections were stained 


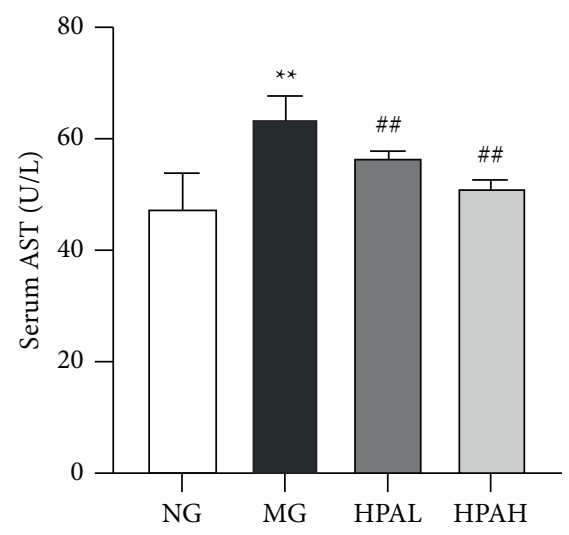

(a)

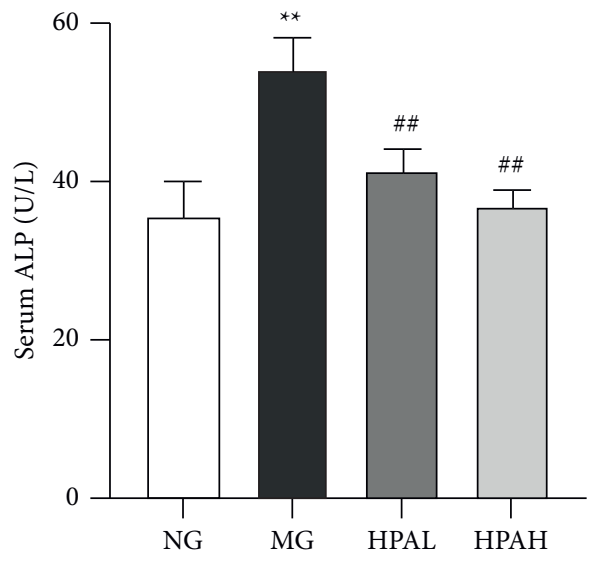

(c)

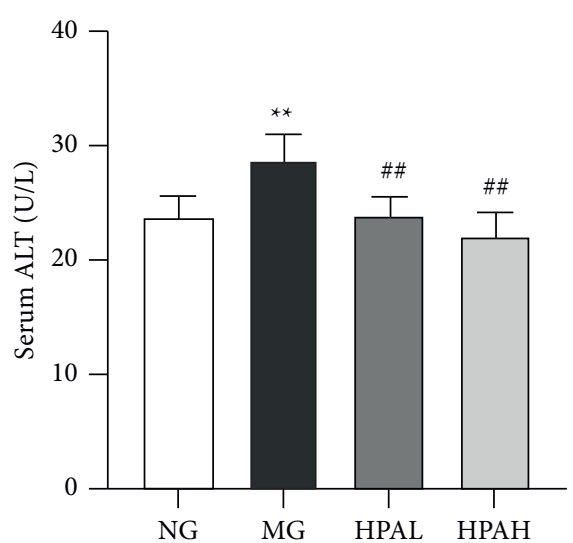

(b)

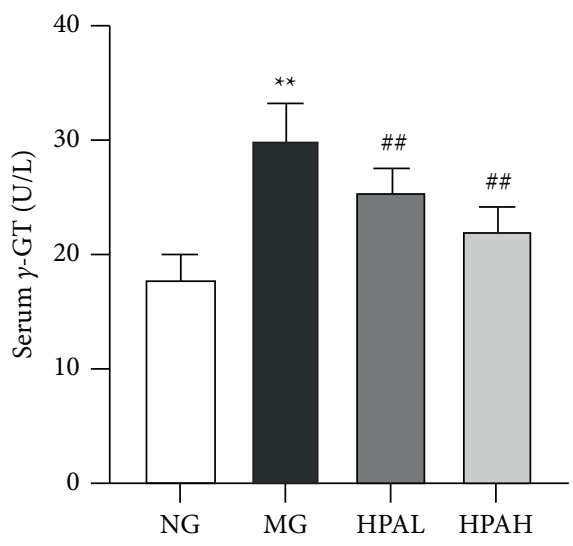

(d)

FIgUre 2: Effect of HPA on the hepatic function in ALD mice. (a) Serum AST; (b) serum ALT; (c) serum ALP; (d) serum $\gamma$-GT. Data were expressed as mean $\pm \mathrm{SD}(n=12) .{ }^{* *} p<0.01$, versus NG; ${ }^{\#} p<0.05$ and ${ }^{\# \#} p<0.01$, versus MG.

by $H \& E$ and Masson and pathological observations were performed. As presented in Figure 6, the structure of hepatocytes and the arrangement of hepatic cords in the NG were normal, and there was no edema and inflammatory infiltration. At the same time, there was no accumulation of collagen fibers in liver tissue. However, the structure of hepatocytes and hepatic cords of alcohol-fed mice were disordered, and there was obvious inflammatory cell infiltration. At the same time, hepatocytes were accompanied by severe accumulation of collagen fibers. After the intervention of HPA, the structure of hepatocytes and hepatic cords was not arranged neatly; there were a small amount of inflammatory cell infiltration and collagen fibers accumulated. But compared with the MG, it had obvious improvement effect.

3.8. Effects of HPA on the Changes of the Gut Microbiota Composition in ALD Mice. In Figure 7(a), the Venn chart shows that the NG, MG, HPAL, and HPAH had a total of 104 OTUs at the genus level. Figures 7(b) and 7(c) show the composition of intestinal flora in each group of mice at the genus and phylum levels. At the genus level (Figure $7(\mathrm{c})$ ), the dominant bacteria in the NG mice were
norank_f_Muribaculaceae and Lactobacillus, accounting for $29.36 \%$ and $21.25 \%$ respectively. While, in the MG, the dominant bacteria were Lactobacillus (35.24\%), norank_f_Muribaculaceae (25.77\%), and Faecalibaculum (5.80\%). After being treated with HPA, the abundance of Feacalibaculum and norank_f_Muribaculaceae were decreased to some extent, and the abundance of Prevotellaceae was increased (versus MG). At the phylum level (Figure 7(b)), the abundance of Firmicutes in MG was significantly increased, and the content of Bacteroidota was decreased (versus NG). Compared with the MG, the abundance of Firmicutes was decreased, and the Verrucomicrobiota was increased.

The correlation between gut microbial flora and biochemical indicators is shown in Figure 7(d). There were both positive and negative correlations between serum lipids indexes and each colony. The Romboutsia was positively correlated with AST and hepatic TC $(p \leq 0.001)$. The relative abundance of Turicibacter was significantly positively correlated with IL-6, and it was negatively correlated with SOD $(p \leq 0.001)$. Both unclassified_k_norank_d_Bacteria and Bifidobacterium showed positive correlation with serum LDL-C, while norank_f_Erysipelotrichpaceae, norank_$f$ _Lachnospiraceae, and Colidextribacter were negatively 


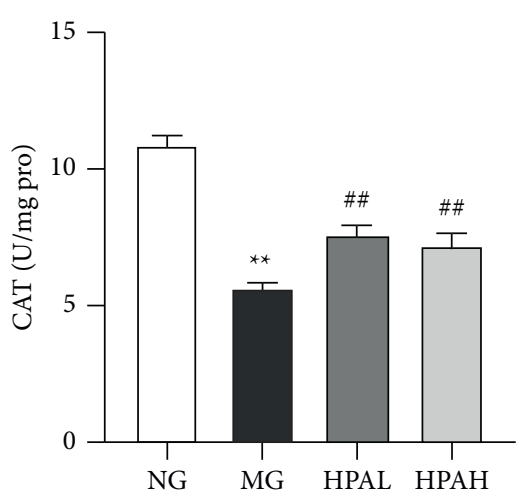

(a)

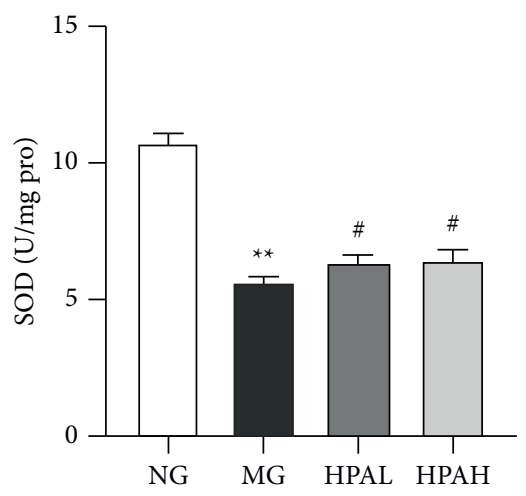

(b)

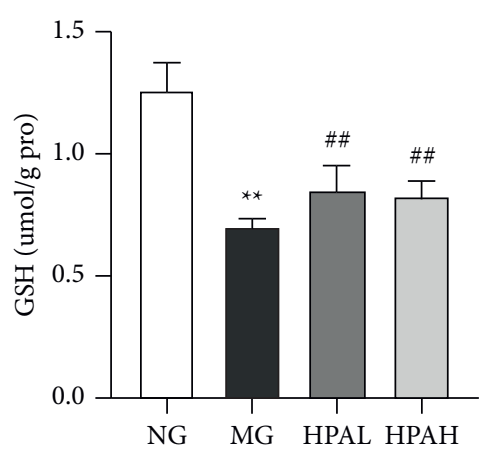

(c)

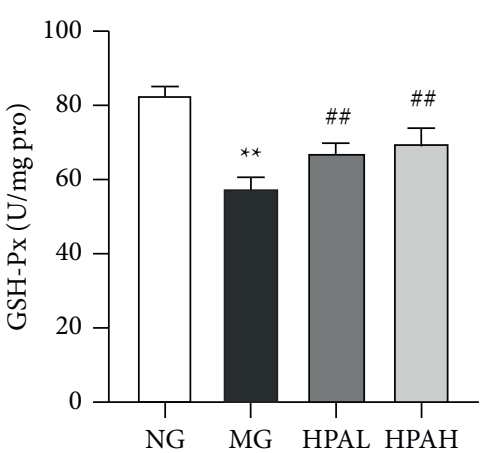

(d)

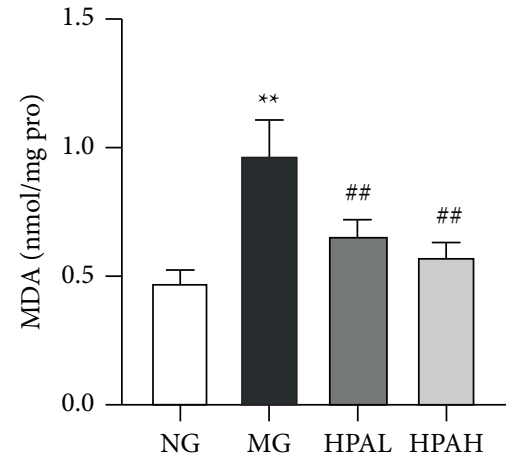

(e)

FIgURE 3: Effect of HPA on the antioxidant capacities in ALD mice. (a) CAT activity; (b) SOD activity; (c) GSH activity; (d) GSH-Px activity; (e) MDA level. Data were expressed as mean \pm SD $(n=12) .{ }^{* *} p<0.01$, versus NG; ${ }^{\#} p<0.05$ and ${ }^{\# \#} p<0.01$, versus MG.

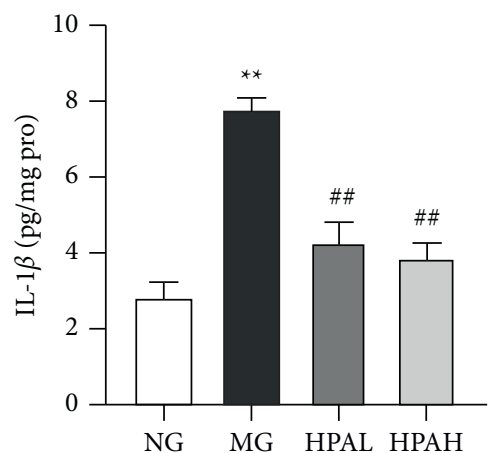

(a)

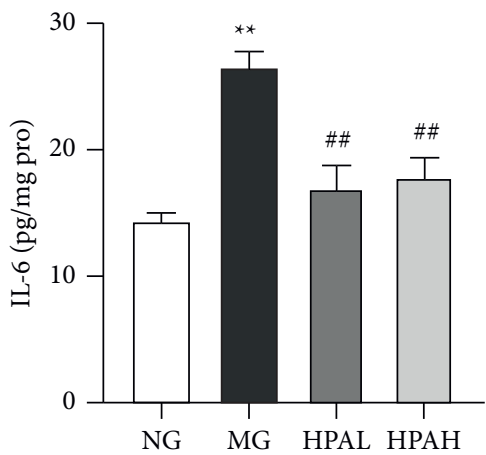

(b)

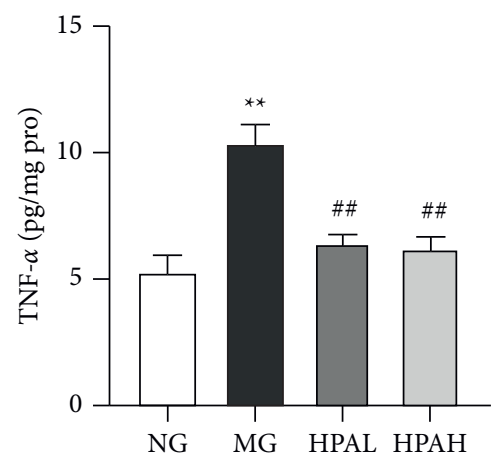

(c)

FIgURE 4: Effect of HPA on inflammation response in ALD mice. (a) Hepatic IL-1 $\beta$; (b) hepatic IL-6; (c) hepatic TNG- $\alpha$. Data were expressed as mean $\pm \mathrm{SD}(n=12)$. ${ }^{* *} p<0.01$, versus NG; ${ }^{\#} p<0.05$ and ${ }^{\# \#} p<0.01$, versus MG.

correlated with serum LDL-C $(p \leq 0.001)$. Colidextribacter was negatively correlated with TNF- $\alpha$ and hepatic FFA $(p \leq 0.001)$. The relative abundance of Roseburia was obviously negatively correlated with ALP $(p \leq 0.001)$.

\section{Discussion}

ALD is the leading cause of global liver disease, and its risk increases with increased dose and duration of alcohol consumption $[22,23]$. Some scholars have pointed out that oxidative stress and inflammatory cytokines could directly or indirectly make the development of ALD more serious $[24,25]$. In this study, the alcohol-induced ALD mice model was established. The purpose of experiment was to explain the mechanism of HPA to improve ALD in mice by studying detected liver index, blood lipids, oxidation markers, and inflammatory factors. In addition, the regulatory effect of HPA on intestinal microflora in ALD mice was also investigated.

The final weight, food intake, and hepatic index in the MG were significantly different from those in the NG (Table 1). After treatment with high-dose HPA, the liver 


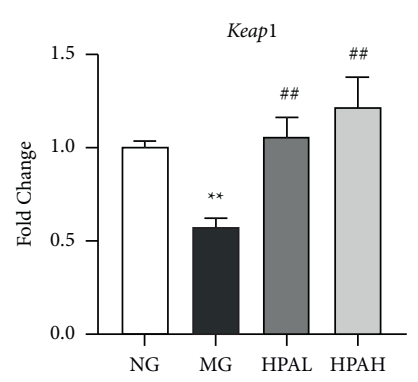

(a)

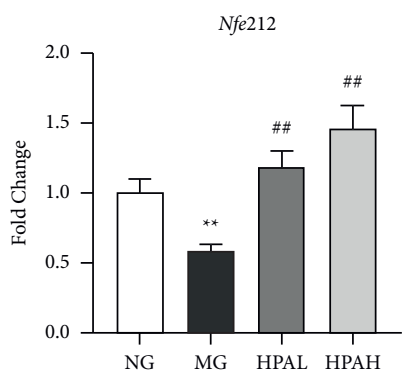

(b)

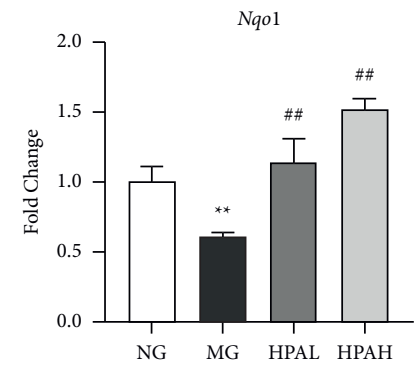

(c)

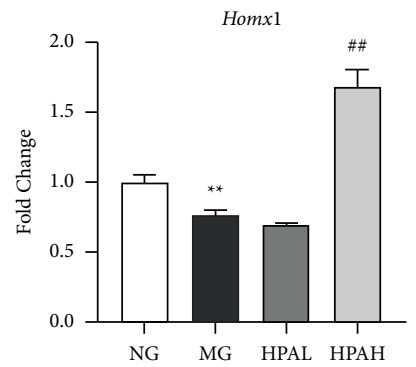

(d)

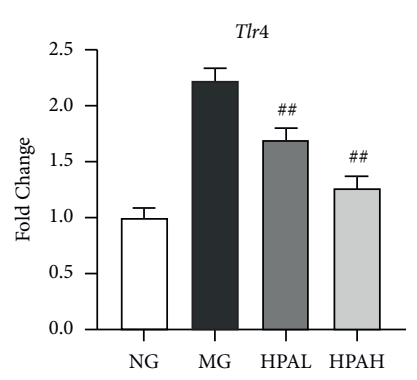

(e)

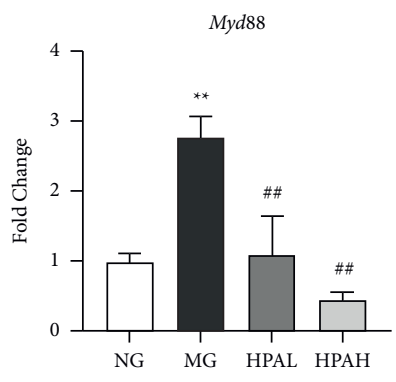

(f)

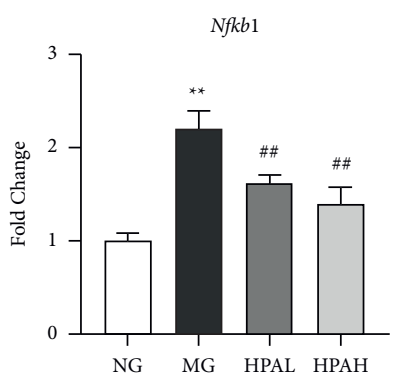

(g)

Figure 5: Effect of HPA on oxidative stress and inflammation-related gene expression in ALD mice. (a) Keap1; (b) Nfe2l2; (c) Nqo1; (d) Homx1; (e) Tlr4; (f) Myd88; (g) Nfkb1. Data were expressed as mean \pm SD $(n=12) .{ }^{* *} p<0.01$, versus NG; ${ }^{\#} p<0.01$, versus MG.
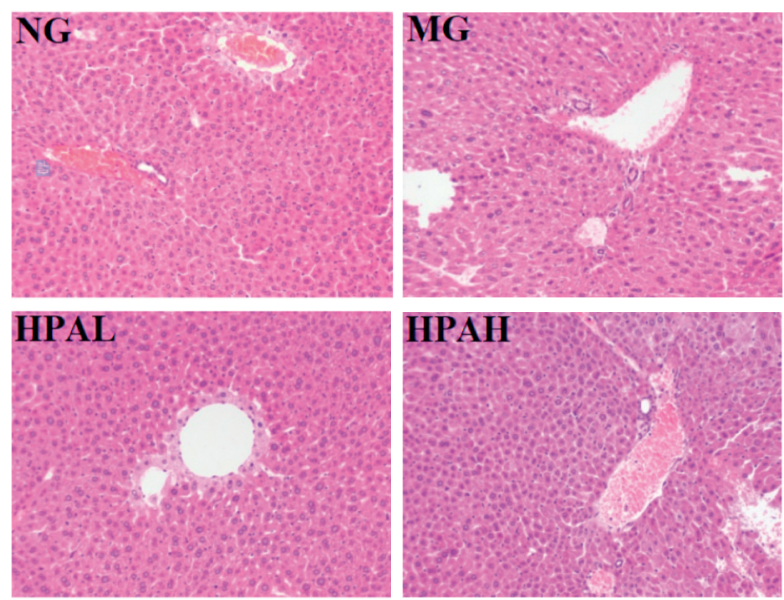

(a)
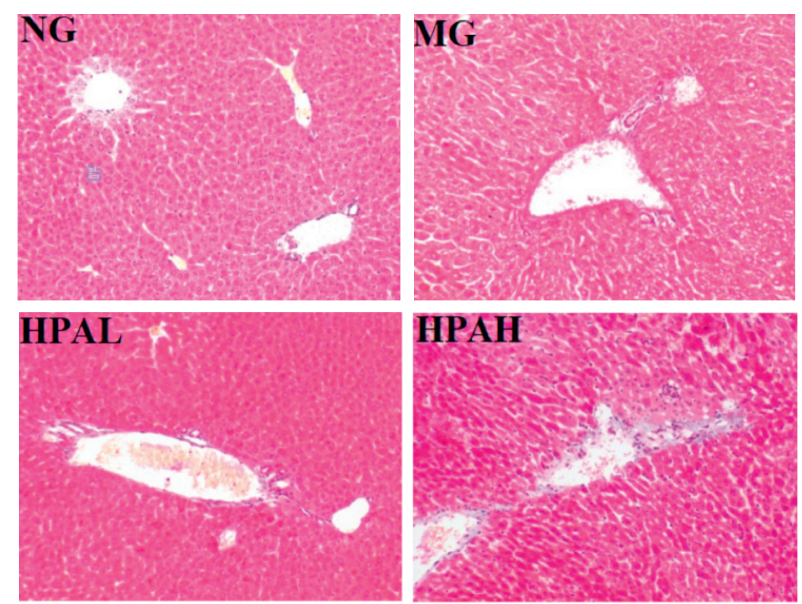

(b)

FIgURE 6: Histopathological detection of livers in ALD mice. (a) H\&E staining in liver ( $\times 200$ magnification); (b) Masson staining in liver (×200 magnification).

index was obviously reduced (versus MG). However, there was no significant change in these indexes between the HPAL and the MG. This shows that the liver index decreases with the increase of the HPA.

Some studies have shown that alcoholic liver injury could cause excessive accumulation of lipids in serum and liver, resulting in dyslipidemia in the body and the formation of alcoholic fatty liver $[26,27]$. The current results showed that both high-dose and low-dose HPA could significantly improve the abnormalities of serum lipids and liver lipids (TC, TG, and FFA) caused by alcohol (versus MG, Figures $1(\mathrm{a})-1(\mathrm{~h}))$. HPA could obviously reduce the content of LDL-C and increased the content of HDL-C; this result was consistent with the study of Sadeghi et al. [28]. Highdose HPA had the best effect in improving blood lipids.

The activities of AST, ALT, ALP, and $\gamma$-GT will increase with alcohol intake, thus aggravating the degree of liver injury, so they are important indicators for evaluating liver function $[29,30]$. The results showed that all treatment groups could clearly reduce the levels of the above four indexes (Figure 2). Therefore, HPA could improve liver dysfunction cause by alcohol, and the improvement effect of high-dose HPA was the best.

Oxidative stress refers to the imbalance between reactive oxygen species and antioxidants, which can be exacerbated by excessive alcohol intake $[25,31]$. In a normal 


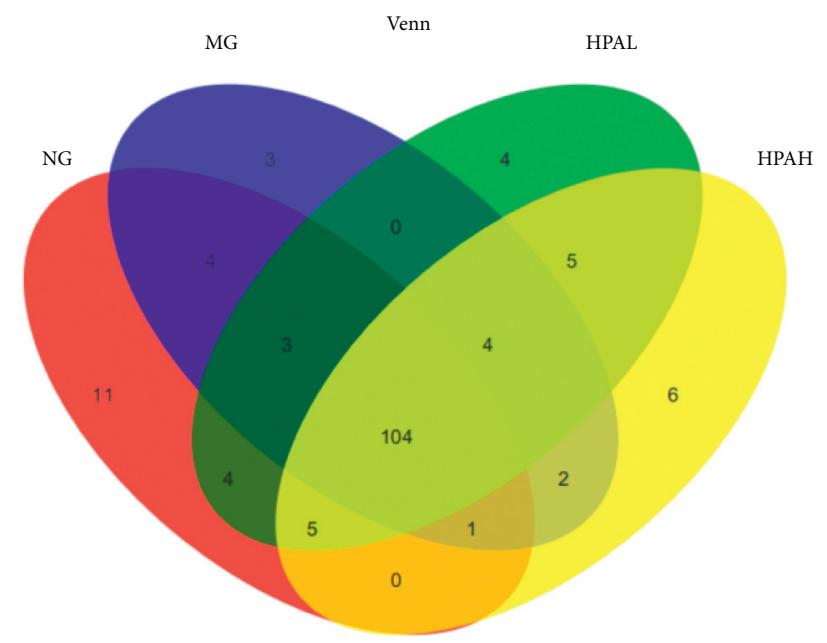

(a)

Community barplot analysis

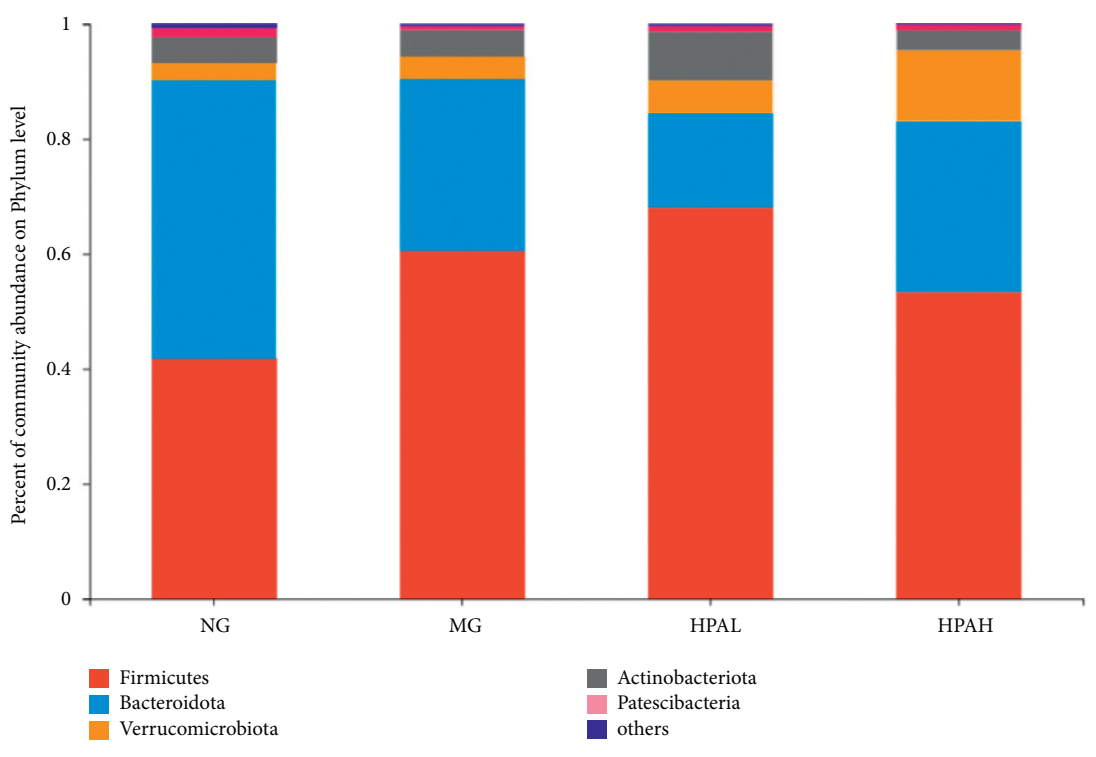

(b)

Figure 7: Continued. 
Community barplot analysis

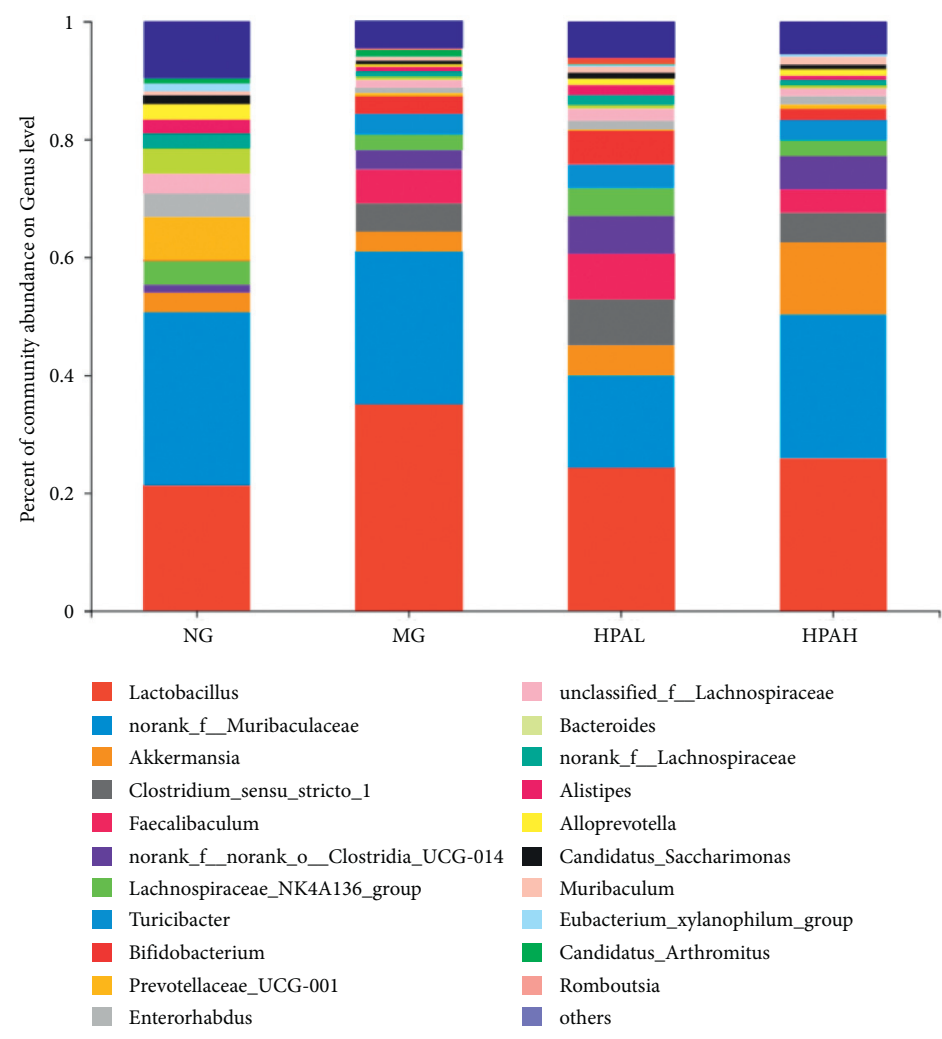

(c)

Figure 7: Continued. 

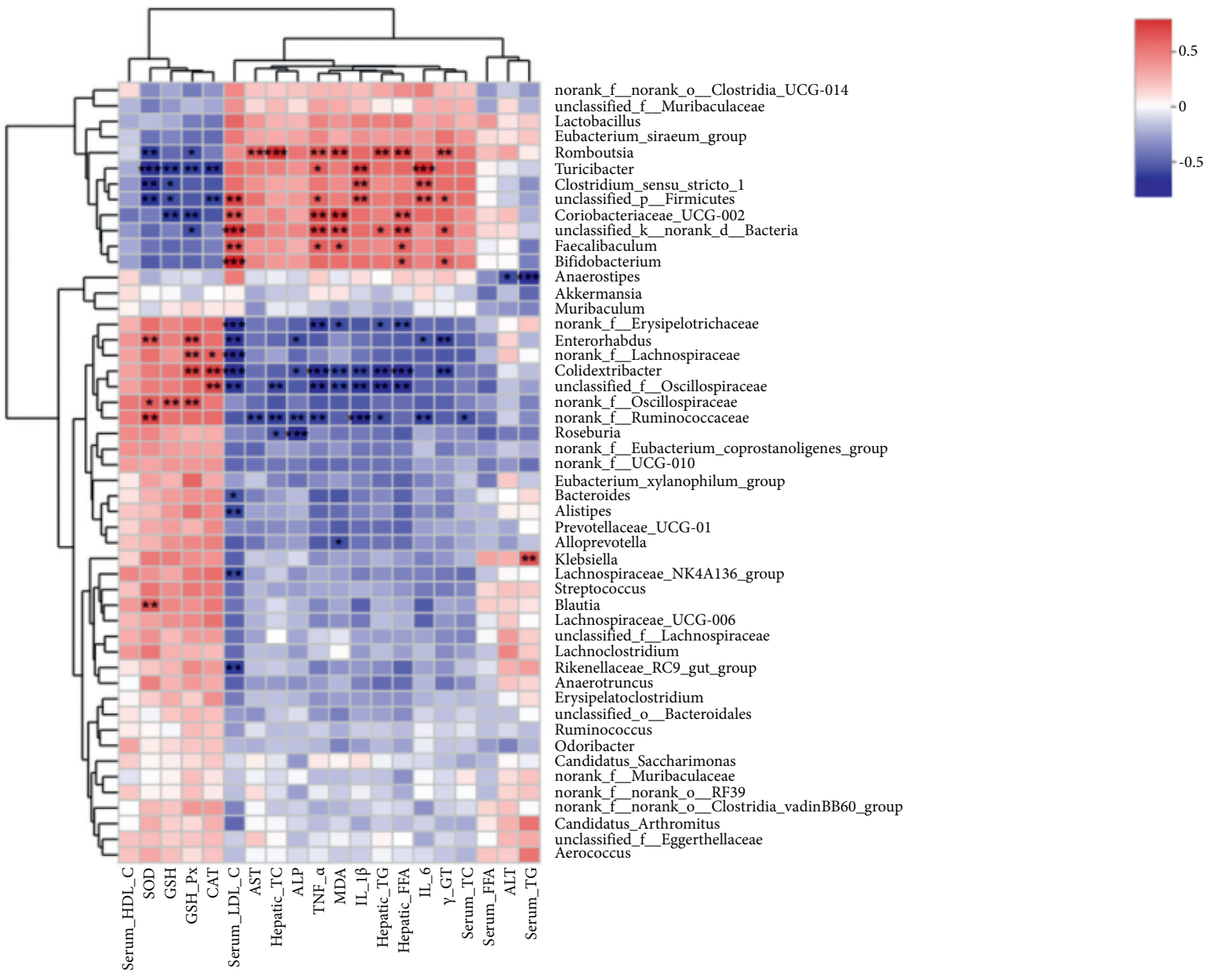

(d)

FIGURE 7: Effects of HPA on the changes of the colonic microbiota composition in ALD mice. (a) Venn diagrams of HPA; (b) percent of community abundance at phylum level; (c) percent of community abundance at genus level; (d) heatmap of spearman's correlation analysis of the biological parameters and relative abundance of colonic microbiota at species level. ${ }^{* * *} p \leq 0.001$ and ${ }^{* *} p \leq 0.01$.

physiological environment, antioxidants are present in human cells to eliminate harmful free radicals, such as CAT, SOD, and GSH [32]. KEAP1/NRF2 is a classical oxidative stress pathway, in which NRF2 is a key transcription factor in the process of oxidative stress [33, 34]. After NFR2 is isolated into the nucleus, it binds to the ARE site to activate the expression of downstream Hmox-1 and Nqo1 gene, thus increasing the production of antioxidant enzymes and regulating the level of oxidative stress [35]. Figure 3 shows that the levels of oxidative stress markers were significantly increased in all treatment groups (versus MG). Interestingly, the low-dose HPA had a good effect on CAT and GSH levels; the high-dose HPA had the good effect on SOD and GSH-Px. In addition, HPA could obviously reduce the level of MDA in alcohol-treated mice (versus MG). At the genetic level, HPA could apparently enhance the expression of antioxidant genes (Figures 5(a)$5(d))$, so as to improve the effects on oxidative stress caused by ALD.
Continuous intake of alcohol activates proinflammatory cytokines, leading to liver inflammation [36]. When NF- $\kappa \mathrm{B}$ is transferred from the cytoplasm to the nucleus, it releases inflammatory mediators, including IL- $1 \beta$, IL-6, and TNF- $\alpha$ [37]. These inflammatory mediators disrupt the balance between proinflammatory and anti-inflammatory factors, so they play an important role in the pathogenesis of ALD [38]. Excessive ethanol intake will increase the level of toxin in the body; the TLR4 was activated and then the expression level of $M y d 88$ gene signal pathway was increased, leading to liver inflammation [39]. In particular, the levels of IL-1 $\beta$, IL-6, and TNF- $\alpha$ in alcohol-treated mice decreased significantly after HPA treatment (Figures 4(a)-4(c)). In the report of Liao et al. [17], HPA also reduced the inflammatory cytokines in alcohol-induced ALD mice, which is consistent with our results. In Figures 6(a) and 6(b), the expression of genes related to inflammation was decreased in all treatment groups, and the improvement effect of high-dose HPA was the best. 
It has been reported that long-term alcohol intake can lead to a disorder in the abundance of intestinal microflora [30]. In the intestinal microflora, there have been beneficial and harmful bacteria. Among them, Faecalibaculum is a representative genus of intestinal bacteria in alcoholic liver injury, which has the effect of promoting inflammation [40]. norank $f \_$Muribaculaceae has a great relationship with human obesity; the higher its abundance is, the thinner the person is [41]. Akkermansia can reduce intestinal permeability, and as its abundance increases, so does the content of lipopolysaccharide [42]. In this experiment, the results showed that the abundance of harmful bacteria (norank_f_Muribaculaceae, Faecalibaculum, and unclassified_p_Firmicutes) in alcohol-treated mice was increased. After treatment with HPA, the harmful bacteria abundance was decreased, and the beneficial bacteria (Akkermansia) was increased. The results showed that HPA could improve the intestinal microbial disorder caused by alcohol by changing the abundance of intestinal flora.

In this study, we found that HPA could reduce the indexes of serum and liver lipid in alcohol-treated mice. HPA could improve liver inflammation and oxidative stress in ALD mice by promoting or inhibiting the expression of inflammatory factors and antioxidative stress genes. After the ALD mice were treatment by HPA, the beneficial bacteria were increased and harmful bacteria were reduced.

\section{Conclusion}

In general, HPA could improve the lipid accumulation in serum and liver caused by alcohol and also inhibit the development of inflammatory factors and liver fibrosis. On the contrary, HPA could improve the activities of liver function and oxidation markers and regulate the imbalance of intestinal microflora. Quantitative RT-PCR results showed that HPA could alleviate oxidative stress and inflammation of ALD through two signal pathways: KEAP1/NRF2 and TLR4/MyD88/NF- $\kappa$ B. In addition, high-dose HPA showed a more prominent effect. This project provided a theoretical basis for HPA in the treatment of alcoholic liver disease. At the same time, it provides a certain theoretical basis for the subsequent exploration of the functions of the monomer components in HPA. It also provides new ideas for the treatment or prevention of ALD. In the future, we will study the effective monomer components in HPA.

\section{Data Availability}

The data used to support the findings of this study are available from the corresponding author upon request.

\section{Disclosure}

Jingxuan Zhou and Nanhai Zhang share first authorship.

\section{Conflicts of Interest}

The authors declare that the research was conducted in the absence of any commercial or financial relationships that could be construed as potential conflicts of interest.

\section{Authors' Contributions}

JZ and NZ performed the experiments and wrote the manuscript. LZhao revised the manuscript. WW and JL supervised the project. FZ and LZhan contributed to conception and design the project. FZ, JL, LZhan, and M.M. Soliman contributed to acquisition of the financial support for the project. All authors have read and agreed to the published version of the manuscript. Jingxuan Zhou and Nanhai Zhang have contributed equally to this work.

\section{Acknowledgments}

This study was financially supported by the Deep Process and Functional Food Development of Daylily and Astragalus (no. 201904710611637), Taif University Researchers Supporting Project (TURSP-2020/09), and the National Dairy Industry and Technology System of China (Grant no. CARS36).

\section{References}

[1] J. Rehm, A. V. Samokhvalov, and K. D. Shield, "Global burden of alcoholic liver diseases," Journal of Hepatology, vol. 59, no. 1, pp. 160-168, 2013.

[2] J. S. Bajaj, "Alcohol, liver disease and the gut microbiota," Nature Reviews Gastroenterology \& Hepatology, vol. 16, no. 4, pp. 235-246, 2019.

[3] P. Mathurin and R. Bataller, "Trends in the management and burden of alcoholic liver disease," Journal of Hepatology, vol. 62, no. 1, pp. S38-S46, 2015.

[4] Y. Lv, K. F. So, and J. Xiao, "Liver regeneration and alcoholic liver disease," Annals of Translational Medicine, vol. 8, no. 8, Article ID 567, 2020.

[5] L. Zhao, A. Mehmood, D. Yuan et al., "Protective mechanism of edible food plants against alcoholic liver disease with special mention to polyphenolic compounds," Nutrients, vol. 13, no. 5, Article ID 1612, 2021.

[6] V. Rosato, L. Abenavoli, A. Federico, M. Masarone, and M. Persico, "Pharmacotherapy of alcoholic liver disease in clinical practice," International Journal of Clinical Practice, vol. 70, no. 2, pp. 119-131, 2016.

[7] A. K. Singal, R. Bataller, J. Ahn, P. S. Kamath, and V. H. Shah, "ACG clinical guideline: alcoholic liver disease," American Journal of Gastroenterology, vol. 113, no. 2, pp. 175-194, 2018.

[8] K. K. Auyeung, Q.-B. Han, and J. K. Ko, “Astragalus membranaceus: a Review of its protection against inflammation and gastrointestinal cancers," The American Journal of Chinese Medicine, vol. 44, no. 1, pp. 1-22, 2016.

[9] T. Nozaki, J. Minaguchi, K. Takehana, and H. Ueda, "Antidiabetic activities of traditional Chinese herbal medicine in streptozotocin-induced diabetic rats," Okajimas Folia Anatomica Japonica, vol. 93, no. 4, pp. 111-118, 2017.

[10] L. Yang, X. Han, F. Xing et al., "Total flavonoids of astragalus attenuates experimental autoimmune encephalomyelitis by suppressing the activation and inflammatory responses of microglia via JNK/AKT/NF $\kappa$ B signaling pathway," Phytomedicine, vol. 80, Article ID 153385, 2021.

[11] W.-Y. Sun, W. Wei, S.-Y. Gui, L. Wu, and H. Wang, "Protective effect of extract fromPaeonia lactifloraandAstragalus membranaceusagainst liver injury induced by Bacillus calmette-guérin and lipopolysaccharide in mice," Basic and 
Clinical Pharmacology and Toxicology, vol. 103, no. 2, pp. 143-149, 2008.

[12] J. Zhou, N. Zhang, L. Zhao et al., "Astragalus polysaccharides and saponins alleviate liver injury and regulate gut microbiota in alcohol liver disease mice," Foods, vol. 10, no. 11, Article ID 2688, 2021.

[13] N. Ramli, K.-Y. Chin, K. Zarkasi, and F. Ahmad, "A review on the protective effects of honey against metabolic syndrome," Nutrients, vol. 10, no. 8, Article ID 1009, 2018.

[14] H. Laaroussi, M. Bakour, D. Ousaaid et al., "Protective effect of honey and propolis against gentamicin-induced oxidative stress and hepatorenal damages," Oxidative Medicine and Cellular Longevity, vol. 2021, Article ID 9719906, 19 pages, 2021.

[15] N. S. Nayan, M. A. M. Yazid, K. Nallappan et al., "In vitro modulation of endogenous antioxidant enzyme activities and oxidative stress in autism lymphoblastoid cell line (ALCL) by stingless bee honey treatment," Oxidative Medicine and Cellular Longevity, vol. 2020, Article ID 4539891, 7 pages, 2020.

[16] M. Xiao, H. Chen, Z. Shi, Y. Feng, and W. Rui, "Rapid and reliable method for analysis of raw and honey-processed astragalus by UPLC/ESI-Q-TOF-MS using HSS T3 columns," Analytical Methods, vol. 6, no. 19, pp. 8045-8054, 2014.

[17] J. Liao, C. Li, J. Huang et al., "Structure characterization of honey-processed astragalus polysaccharides and its anti-inflammatory activity in vitro," Molecules, vol. 23, no. 1, Article ID 168, 2018.

[18] J. Wu, C. Li, L. Bai et al., "Structural differences of polysaccharides from Astragalus before and after honey processing and their effects on colitis mice," International Journal of Biological Macromolecules, vol. 182, pp. 815-824, 2021.

[19] L. Zhao, N. Zhang, D. Yang et al., "Protective effects of five structurally diverse flavonoid subgroups against chronic alcohol-induced hepatic damage in a mouse model," Nutrients, vol. 10, no. 11, Article ID 1754, 2018.

[20] H.-Y. Koo, M. A. Wallig, B. H. Chung, T. Y. Nara, B. H. S. Cho, and M. T. Nakamura, "Dietary fructose induces a wide range of genes with distinct shift in carbohydrate and lipid metabolism in fed and fasted rat liver," Biochimica et Biophysica Acta-Molecular Basis of Disease, vol. 1782, no. 5, pp. 341-348, 2008.

[21] S. C. Taylor, K. Nadeau, M. Abbasi, C. lachance, M. Nguyen, and J. Fenrich, "The ultimate qPCR experiment: producing publication quality, reproducible data the first time," Trends in Biotechnology, vol. 37, no. 7, pp. 761-774, 2019.

[22] G. E. Arteel, "Liver-lung axes in alcohol-related liver disease," Clinical and Molecular Hepatology, vol. 26, no. 4, pp. 670-676, 2020.

[23] X. Chi, C. Q. Pan, S. Liu, D. Cheng, Z. Cao, and H. Xing, "Regulating intestinal microbiota in the prevention and treatment of alcohol-related liver disease," Canadian Journal of Gastroenterology and Hepatology, vol. 2020, Article ID 6629196, 10 pages, 2020.

[24] H. Kawaratani, T. Tsujimoto, A. Douhara et al., "The effect of inflammatory cytokines in alcoholic liver disease," Mediators of Inflammation, vol. 2013, Article ID 495156, 10 pages, 2013.

[25] H. K. Tan, E. Yates, K. Lilly, and A. D. Dhanda, "Oxidative stress in alcohol-related liver disease," World Journal of Hepatology, vol. 12, no. 7, pp. 332-349, 2020.

[26] H. Chen, F. Shen, A. Sherban et al., "DEP domain-containing mTOR-interacting protein suppresses lipogenesis and ameliorates hepatic steatosis and acute-on-chronic liver injury in alcoholic liver disease," Hepatology, vol. 68, no. 2, pp. 496-514, 2018.

[27] Y. Song, X. Wu, D. Yang et al., "Protective effect of andrographolide on alleviating chronic alcoholic liver disease in mice by inhibiting nuclear factor kappa B and tumor necrosis factor Alpha activation," Journal of Medicinal Food, vol. 23, no. 4, pp. 409-415, 2020.

[28] F. Sadeghi, M. Akhlaghi, and S. Salehi, "Adverse effects of honey on low-density lipoprotein cholesterol and adiponectin concentrations in patients with type 2 diabetes: a randomized controlled cross-over trial," Journal of Diabetes and Metabolic Disorders, vol. 19, no. 1, pp. 373-380, 2020.

[29] Y. Sutoh, T. Hachiya, Y. Suzuki et al., "ALDH2 genotype modulates the association between alcohol consumption and AST/ALT ratio among middle-aged Japanese men: a genomewide G $\times$ E interaction analysis," Scientific Reports, vol. 10, no. 1, Article ID 16227, 2020.

[30] M. Zahid, M. Arif, M. A. Rahman, and M. Mujahid, "Hepatoprotective and antioxidant activities of Annona squamosa seed extract against alcohol-induced liver injury in Sprague Dawley rats," Drug and Chemical Toxicology, vol. 43, no. 6, pp. 588-594, 2020.

[31] A. Michalak, T. Lach, and H. Cichoż-Lach, "Oxidative stress-a key player in the course of alcohol-related liver disease," Journal of Clinical Medicine, vol. 10, no. 14, Article ID 3011, 2021.

[32] L. Zhao, A. Mehmood, M. M. Soliman et al., "Protective effects of ellagic acid against alcoholic liver disease in mice," Frontiers in nutrition, vol. 8, Article ID 744520, 2021.

[33] J. Sun, Z. Hong, S. Shao et al., "Liver-specific Nrf2 deficiency accelerates ethanol-induced lethality and hepatic injury in vivo," Toxicology and Applied Pharmacology, vol. 426, Article ID 115617, 2021.

[34] M. Yamamoto, T. W. Kensler, and H. Motohashi, "The KEAP1-NRF2 System: a thiol-based sensor-effector apparatus for maintaining redox homeostasis," Physiological Reviews, vol. 98, no. 3, pp. 1169-1203, 2018.

[35] A. Loboda, M. Damulewicz, E. Pyza, A. Jozkowicz, and J. Dulak, "Role of Nrf2/HO-1 system in development, oxidative stress response and diseases: an evolutionarily conserved mechanism," Cellular and Molecular Life Sciences, vol. 73, no. 17, pp. 3221-3247, 2016.

[36] N. Hosseini, J. Shor, and G. Szabo, "Alcoholic hepatitis: a review," Alcohol and Alcoholism, vol. 54, no. 4, pp. 408-416, 2019.

[37] Q.-H. Huang, L.-Q. Xu, Y.-H. Liu et al., "Polydatin protects rat liver against ethanol-induced injury: involvement of CYP2E1/ROS/Nrf2 and TLR4/NF- $\kappa$ B p65 pathway," Evidence-based Complementary and Alternative Medicine, vol. 2017, Article ID 7953850, 14 pages, 2017.

[38] B. Gao, "Hepatoprotective and anti-inflammatory cytokines in alcoholic liver disease," Journal of Gastroenterology and Hepatology, vol. 27, pp. 89-93, 2012.

[39] S. Patel, R. Behara, G. Swanson, C. Forsyth, R. Voigt, and A. Keshavarzian, "Alcohol and the intestine," Biomolecules, vol. 5, no. 4, pp. 2573-2588, 2015.

[40] V. B. Dubinkina, A. V. Tyakht, V. Y. Odintsova et al., "Links of gut microbiota composition with alcohol dependence syndrome and alcoholic liver disease," Microbiome, vol. 5, no. 1, Article ID 141, 2017.

[41] D. Hou, Q. Zhao, L. Yousaf, J. Khan, Y. Xue, and Q. Shen, "Consumption of mung bean (Vigna radiata L.) attenuates obesity, ameliorates lipid metabolic disorders and modifies the gut microbiota composition in mice fed a high-fat diet," Journal of Functional Foods, vol. 64, Article ID 103687, 2020.

[42] J. Fan, Y. Wang, Y. You et al., "Fermented ginseng improved alcohol liver injury in association with changes in the gut microbiota of mice," Food \& Function, vol. 10, no. 9, pp. 5566-5573, 2019. 